\title{
The Role of FTO and Vitamin D for the Weight Loss Effect of Roux-en-Y Gastric Bypass Surgery in Obese Patients
}

\author{
Marcus Bandstein • Bernd Schultes • Barbara Ernst • \\ Martin Thurnheer • Helgi B. Schiöth • Christian Benedict
}

Published online: 28 February 2015

(C) The Author(s) 2015. This article is published with open access at Springerlink.com

\begin{abstract}
Background A recent study in children demonstrated that the rs9939609 single-nucleotide polymorphism in the fat mass and obesity $(F T O)$ gene influences prospective weight gain, however, only in those who were vitamin D-deficient. If this might also be the case for Roux-en-Y gastric bypass (RYGB), surgery-induced weight loss is however unknown. The objective of this study is to examine if the magnitude of RYGB surgery-induced weight loss after 2 years depends on patients' FTO rs9939609 genotype (i.e., TT, AT, and AA) and presurgery vitamin $\mathrm{D}$ status ( $<50 \mathrm{nmol} / \mathrm{L}$ equals deficiency).

Methods Before and at 24 months after RYGB surgery, BMI was measured in 210 obese patients (mean BMI $45 \mathrm{~kg} / \mathrm{m}^{2}$, $72 \%$ females). Serum 25 -hydroxyvitamin D3 levels were also repeatedly measured. Following surgery, vitamin D was supplemented. Possible weight loss differences between genotypes were tested with multiple linear regressions.
\end{abstract}

Helgi B. Schiöth and Christian Benedict have equal contributions.

M. Bandstein $(\varangle) \cdot$ H. B. Schiöth • C. Benedict

Department Neuroscience, Functional Pharmacology, Uppsala

University, Box 593, 75124 Uppsala, Sweden

e-mail: marcus.bandstein@neuro.uu.se

H. B. Schiöth

e-mail: helgi.schioth@neuro.uu.se

C. Benedict

e-mail: christian.benedict@neuro.uu.se

B. Schultes $\cdot$ B. Ernst $\cdot$ M. Thurnheer

Interdisciplinary Obesity Center, eSwiss Medical \& Surgical Center,

St. Gallen, Switzerland

B. Schultes

e-mail: bernd.schultes@stephanshorn.ch

B. Ernst

e-mail: barbara.ernst@stephanshorn.ch

M. Thurnheer

e-mail: martin.thurnheer@stephanshorn.ch
Results The per-allele effect of each FTO A-allele on excessive BMI loss (EBMIL) was $3 \%(P=0.02)$. When split by baseline status, the EBMIL of vitamin D-deficient patients carrying AA exceeded that of vitamin D-deficient patients carrying TT by $\sim 14 \%(P=0.03)$. No such genotypic differences were found in patients without presurgery vitamin $D$ deficiency. Post-surgery serum levels of vitamin D did not differ between groups.

Conclusions Our data suggest that presurgery vitamin D levels influence the size of genotype effects of FTO rs9939609 on RYGB surgery-induced weight loss in obese patients.

Keywords Vitamin D · FTO $\cdot$ RYGB $\cdot$ Weight loss $\cdot$ Bariatric surgery

\section{Introduction}

Large cross-sectional studies have demonstrated that both children and adults carrying the rs9939609 obesity A-allele within an intron region of the fat- and obesity-associated gene (FTO) exhibit higher BMI scores and are more often obese than noncarriers [1-4]. In addition, a recent study involving 1 , 088 children demonstrated that carriers of the FTO A-allele gained more weight than noncarriers during a $\sim 5$-year observation period [5]. Complementing its important role for human body weight dynamics, some studies have demonstrated that FTO rs9939609 (or proxy single-nucleotide polymorphisms) could also partially account for interindividual differences in weight loss upon Roux-en-Y gastric bypass (RYGB) surgery [6-8]. Most interestingly, in the aforementioned study investigating the impact of FTO on weight gain during childhood, genotypic differences in weight gain were only seen between vitamin D-deficient children [5]. This suggests that 
the magnitude by which FTO influences human weight regulation may partially depend on nutritional factors, comprising vitamin $\mathrm{D}$. However, as of yet, no study has investigated if the influence of FTO on RYGB surgery-induced weight loss is modulated by a patient's presurgery vitamin $\mathrm{D}$ status. Thus, the present study aimed at investigating if the FTO A-allele predicts the 2-year weight loss response following RYGB surgery and if this depends on a patient's vitamin D status. Importantly, following surgery, patients received vitamin D supplements to avoid vitamin D undersupply.

\section{Material and Methods}

Patients

RYGB surgeries and follow-up investigations were performed at the Interdisciplinary Obesity Center, St. Gallen, Switzerland. Before surgery, all patients ( $n=210,72 \%$ females) were obese (BMI $45.2 \pm 0.43 \mathrm{~kg} / \mathrm{m}^{2}$ ), and none had undergone any kind of bariatric surgery procedure (e.g., gastric banding) before. Two different variants of RYGB surgery were performed, i.e., proximal and distal RYGBs $[5,9]$. In both procedures, the largest part of the stomach was transected, and a small gastric pouch of about $20-30 \mathrm{~mL}$ was anastomized to the proximal jejunum with the diameter of the pouch-jejunal anastomosis standardized to be about $12 \mathrm{~mm}$. In the proximal RYGB procedure, the biliopancreatic limb was side to side anatomized to the jejunum $150 \mathrm{~cm}$ distal from the pouchjejunal anastomosis (Roux-en-Y limb length, $150 \mathrm{~cm}$ ). In the distal RYGB procedure, the biliopancreatic limb was side to side anatomized to the ileum 60 to $100 \mathrm{~cm}$ proximal from Bauhin's valve (common channel, 60-100 cm). The length to the biliopancreatic limb was approximately $60 \mathrm{~cm}$ in the proximal and 60 to $100 \mathrm{~cm}$ in the distal RYGB procedure.

\section{Assessments}

All participants were genotyped for the $F T O$-associated single-nucleotide polymorphism (SNP) rs9939609 using DNA isolated from whole blood with a custom Illumina iSelect genotyping array (99.5\% success rate). Rs9939609 was confirmed to be in Hardy-Weinberg equilibrium.

At baseline (i.e., close to surgery), blood samples were drawn in the morning (0800-1100h) after an overnight fast, and serum 25-hydroxyvitamin D3 levels were determined by high-performance liquid chromatography (Chromsystems, Instruments \& Chemicals GmbH, Munich, Germany). In general, serum levels of 25-hydroxyvitamin D3 lower than $50 \mathrm{nmol} / \mathrm{L}$ are defined as vitamin D deficiency [10]. Since 25-hydroxyvitamin D3 levels exhibit seasonal variation in obese humans [11], the date where the blood was collected was recorded. Post-surgery, all patients received standard oral vitamin D3 supplements (1,200 IU/day). If serum levels of 25hydroxyvitamin D3 were below $50 \mathrm{nmol} / \mathrm{L}$ at follow-up investigations (i.e., at $+3,+6,+9,+12,+18$, and +24 months), patients received additional intramuscular injection of 300 , 000 IU every 3 months. Further preoperative preparations and post-operative follow-up procedures are described in more detail in [9].

At baseline and 24 months after RYGB surgery, height and weight were measured with patients wearing light clothing and no shoes. BMI was defined as weight $(\mathrm{kg})$ divided by height squared $\left(\mathrm{m}^{2}\right)$. BMI was utilized to calculate relative excessive BMI loss (EBMIL, cutoff for normal-weight $\left.\mathrm{BMI}=25 \mathrm{~kg} / \mathrm{m}^{2}\right)$ by the following equation [12]: $1-(($ initial BMI-final BMI $/($ initial BMI-25)) $) \times 100$.

\section{Statistics}

A univariate general linear model (SPSS Statistics, version 21.0 for Windows, IBM, Chicago, IL, USA) was used to perform the regression analysis (assuming an additive model). Predictors of interest were the three-level FTO rs9939609 genotype $(\mathrm{TT}=0, \mathrm{AT} / \mathrm{TA}=1, \mathrm{AA}=2)$, baseline serum vitamin $\mathrm{D}$ levels, and their interaction term. In case of a significant interaction of FTO with baseline serum vitamin D levels, differences between genotypes were specified by post hoc ANCOVA analysis. Linear mixed effect models were used to investigate if FTO genotype groups, split by baseline vitamin D status, would exhibit post-surgery differences in the time course of serum levels of this micronutrient (i.e., comprising measurements at $3,6,12,18$, to 24 months). All analyses were adjusted for age, sex, and BMI at baseline, unless otherwise specified. Distal RYGB surgery is likely to cause stronger malabsorption of vitamin D than the proximal surgery procedure because the Y-connection is formed much closer to the lower end of the small intestine. Given that the vitamin $\mathrm{D}$ status was a variable of particular interest in our study, we also covaried for the surgery type in our analyses. The date where patients' baseline session took place, comprising the measurement of circulating 25-hydroxyvitamin D3 levels, was utilized as nominal variable in our statistical models to account for seasonal variations of this vitamin (subdivided into six time intervals, i.e., January-February, March-April, May-June, etc.). Overall, a $P$ value less than 0.05 was considered significant. Data are shown as mean \pm SEM, unless otherwise specified.

\section{Results}

As summarized in Table 1, approximately $70 \%$ of our obese patients carried at least one copy of the FTO A-allele. In addition, $\sim 50 \%$ of our obese patients were vitamin D-deficient 
Table 1 Patients' characteristics

\begin{tabular}{|c|c|}
\hline \multicolumn{2}{|l|}{ Sex, $n$ (\% cohort $)$} \\
\hline Female & $151(71.9)$ \\
\hline Male & $59(28.1)$ \\
\hline $\operatorname{Age}^{\mathrm{a}}$ (years) & $42.8 \pm 0.8$ \\
\hline $\mathrm{BMI}^{\mathrm{a}}\left(\mathrm{kg} / \mathrm{m}^{2}\right)$ & $45.2 \pm 0.4$ \\
\hline Waist circumference $^{\mathrm{a}}(\mathrm{cm})$ & $129 \pm 2$ \\
\hline Diabetes $^{\mathrm{a}}, n$ (\% cohort $)$ & $17(8.1)$ \\
\hline Serum triglyceride levels ${ }^{\mathrm{a}}(\mathrm{mmol} / \mathrm{L})$ & $1.8 \pm 0.1$ \\
\hline Serum levels of vitamin $\mathrm{D} 3^{\mathrm{a}}(\mathrm{nmol} / \mathrm{L})$ & $52.9 \pm 1.9$ \\
\hline \multicolumn{2}{|l|}{ Vitamin D deficient, $n$ (\% cohort) } \\
\hline Pre-surgery ${ }^{\mathrm{a}}$ & $104(49.5)$ \\
\hline Post-surgery ${ }^{\mathrm{b}}$ & $18(9.1)$ \\
\hline \multicolumn{2}{|l|}{ FTO rs $9939609, n$ (\% cohort) } \\
\hline $\mathrm{TT}$ & $57(27.4)$ \\
\hline AT & $98(46.7)$ \\
\hline AA & $55(26.2)$ \\
\hline \multicolumn{2}{|l|}{ Surgery type, $n$ ( $\%$ cohort) } \\
\hline Distal RYGB & $158(75.2)$ \\
\hline Proximal RYGB & $52(24.8)$ \\
\hline $\operatorname{BMI}^{\mathrm{b}}\left(\mathrm{kg} / \mathrm{m}^{2}\right)$ & $28.6 \pm 3.9$ \\
\hline EBMIL $^{\mathrm{b}}(\%)$ & $83.4 \pm 1.1$ \\
\hline
\end{tabular}

If not otherwise described, data are mean \pm SEM. Blood to determine serum vitamin D levels and FTO rs9939609 genotype was collected in a fasted state. Serum levels of 25 -hydroxyvitamin D3 lower than $50 \mathrm{nmol} /$ $\mathrm{L}$ were defined as vitamin D deficiency. The presence of diabetes was defined as use of oral hypoglycemic agents or insulin

BMI body mass index, EBMIL excessive BMI loss, SEM standard error of mean, $R Y G B$ Roux-en-Y gastric bypass

${ }^{\text {a }}$ At baseline

${ }^{\mathrm{b}}$ At +24 months post-Roux-en-Y gastric bypass surgery

at baseline. Following RYGB surgery, patients exhibited, on average, an EBMIL of $83 \%$.

Association of FTO and Presurgery Vitamin D Status with 2-Year Weight Loss Induced by RYGB Surgery

A univariate general linear model analysis revealed a positive association between the 2-year EBMIL upon RYGB surgery and FTO rs9939609 genotype (independent variable coefficient $B_{(\mathrm{AA})}=20.7, B_{(\mathrm{TA} / \mathrm{AT})}=13.3, B_{(\mathrm{TT})}=0$ (reference value), $P=0.02)$. The per-allele effect of each FTO A-allele on excessive BMI loss (EBMIL) was $3 \%$ (EBMIL, AA, $86.1 \pm 2.3 \%$; TA/AT, $83.0 \pm 1.7 \%$; TT, $81.5 \pm 2.3 \%$; $P=0.02$ ). The strength of this association, however, was influenced by presurgery serum levels of 25-hydroxyvitamin D3 ( $P=0.04$ for the interaction term "FTO*Vitamin D levels"). When split by their baseline vitamin D status, AA patients who were vitamin Ddeficient exhibited a surgery-induced EBMIL that was $\sim 14 \%$ higher than that of vitamin D-deficient TT carriers $(P=0.03$, Fig. 1, left panel). In contrast, no such genotypic differences

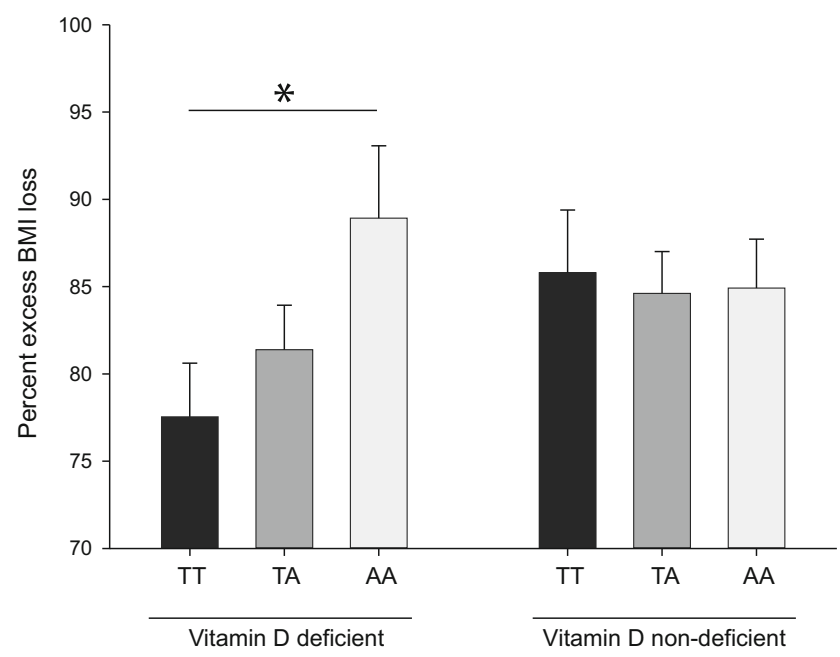

Fig. 1 Percent excess BMI loss at 24 months post-surgery, split by patients' FTO rs9939609 genotype and presurgery vitamin D status. A univariate general linear model was utilized to investigate if the FTO rs9939609 genotype (i.e., AA, AT, or TT) predicts the magnitude of 2year weight loss following Roux-en-Y gastric bypass surgery and if this depends on a patient's baseline vitamin D status (assuming an additive model). Baseline serum levels of 25-hydroxyvitamin D3 $<50 \mathrm{nmol} / \mathrm{L}$ were defined as vitamin D deficiency. Data are shown as mean \pm SEM. ${ }^{*} P<0.05$ for pairwise comparisons between groups

were found in patients without presurgery vitamin $\mathrm{D}$ deficiency $(P=0.89)$, Fig. 1 , right panel). Furthermore, no difference in EBMIL between vitamin D-deficient TT carriers and nondeficient TT carriers was seen $(P=0.23)$. Finally, the presurgery vitamin $\mathrm{D}$ status was not independently linked to EBMIL $(P=0.81)$

Serum Levels of Vitamin D Prior to and After Surgery

Baseline serum levels of 25-hydroxyvitamin D3 revealed that $49.5 \%$ of the patients in our cohort were vitamin D-deficient, compared to $9.1 \%$ who were vitamin D-deficient 2 years after surgery $(P<0.0001)$. A Fisher's exact test showed that serum 25-hydroxyvitamin D3 levels after surgery did not differ between FTO genotype groups, when stratified by baseline vitamin D status $(P>0.05$ at all time points post-surgery; Table 2). As seen in Fig. 2, the mean vitamin D level increased quickly in the vitamin D-deficient groups after surgery (and initiation of vitamin D supplementation). Among the presurgery deficient patients, $30 \%$ remained deficient at the first follow-up visit ( 3 months post-op) and $11 \%$ after 24 months. Some patients with baseline levels above the deficiency cutoff were deficient 24 months after surgery.

\section{Discussion}

Here, we show that patients who were vitamin D-deficient before surgery exhibited a $\sim 14 \%$ higher RYGB surgery- 
Table 2 Number of vitamin D-deficient patients before and at $+3,+6,+$ $9,+12,+18$, and +24 months post-Roux-en-Y gastric bypass surgery, split by FTO rs9939609 genotype and presurgery vitamin D status

\begin{tabular}{|c|c|c|c|c|c|c|c|}
\hline \multirow[t]{2}{*}{ Months } & \multicolumn{3}{|l|}{$n(N)$} & \multicolumn{3}{|l|}{$n(N)$} & \multirow[t]{2}{*}{$P$ value } \\
\hline & $T T^{\mathrm{a}}$ & $T A^{\mathrm{a}}$ & $A A^{\mathrm{a}}$ & $T T$ & $T A$ & $A A$ & \\
\hline Baseline & $35(35)$ & $50(50)$ & $19(19)$ & $0(22)$ & $0(48)$ & $0(36)$ & - \\
\hline+3 & $6(17)$ & $6(24)$ & $2(12)$ & $1(8)$ & $4(25)$ & $4(15)$ & 0.75 \\
\hline+6 & $1(21)$ & $2(30)$ & $1(9)$ & $1(13)$ & $1(30)$ & $1(24)$ & 0.88 \\
\hline+9 & $2(19)$ & $0(32)$ & $1(10)$ & $1(12)$ & 0 (29) & $1(22)$ & 0.09 \\
\hline+12 & $2(22)$ & $2(40)$ & $1(13)$ & $1(13)$ & $1(34)$ & $1(27)$ & 0.84 \\
\hline+18 & $2(23)$ & $3(40)$ & 0 (14) & $2(15)$ & $4(39)$ & $1(25)$ & 0.80 \\
\hline+24 & $5(34)$ & $4(42)$ & $1(18)$ & $2(21)$ & $3(47)$ & $3(35)$ & 0.87 \\
\hline
\end{tabular}

Vitamin D deficiency was defined as serum levels of 25-hydroxyvitamin D3 $<50 \mathrm{nmol} / \mathrm{L}$. Note that following Roux-en-Y gastric bypass surgery, all patients received standard oral vitamin D3 supplements (1,200 IU/ day). If serum levels of 25-hydroxyvitamin D3 were below $50 \mathrm{nmol} / \mathrm{L}$ at follow-up visits, patients received additional intramuscular injection of 300,000 IU every 3 months. $P$ values derive from Fisher's exact test

$n$ number of patients who were vitamin D-deficient, $(N)$ number of patients for whom blood samples were available to determine their serum vitamin D status

${ }^{\text {a } V i t a m i n ~ D-d e f i c i e n t ~ p a t i e n t s ~ b e f o r e ~ s u r g e r y ~(i . e ., ~ a t ~ b a s e l i n e) ~}$

induced weight loss when they carried two copies of the Aallele in the fat mass and obesity (FTO) gene rs9939609, compared to vitamin D-deficient patients who were homozygous for the FTO T-allele. In contrast, no such differences were found between genotypes when presurgery serum vitamin D levels were above $50 \mathrm{nmol} / \mathrm{L}$ (i.e., their vitamin D status was considered sufficient). These results could suggest that presurgery vitamin $\mathrm{D}$ modulates the strength by which FTO predicts RYGB surgery-induced weight loss in obese humans. However, unless independent cohorts can replicate our findings, caution is needed before generalizing our results to patients undergoing other types of gastric bypass surgery (e.g., gastric banding).

Large cross-sectional genome-wide association studies have demonstrated that humans who carry the rs9939609 Aallele have higher BMI scores than noncarriers [1-4]. Each $F T O$ risk allele increases BMI by $0.26-0.66 \mathrm{~kg} / \mathrm{m}^{2}$, equivalent to $\sim 0.84-2.1 \mathrm{~kg}$ in body weight for a 1.80 -m-tall person, and the risk of obesity by $1.25-1.32$ odds [13]. With these findings in mind, it might appear counterintuitive at first glance that our study demonstrates that obese patients who carried the rs9939609 A-allele exhibited the highest EBMIL after RYGB surgery. However, our findings receive also some support from the literature. For instance, in a study involving 520 obese patients, bariatric surgery-induced weight loss was greatest in those who were carrying the rs9939609 A-allele [6]. In addition, a previous nonsurgical study where obese patients participated in a hypocaloric diet demonstrated that a
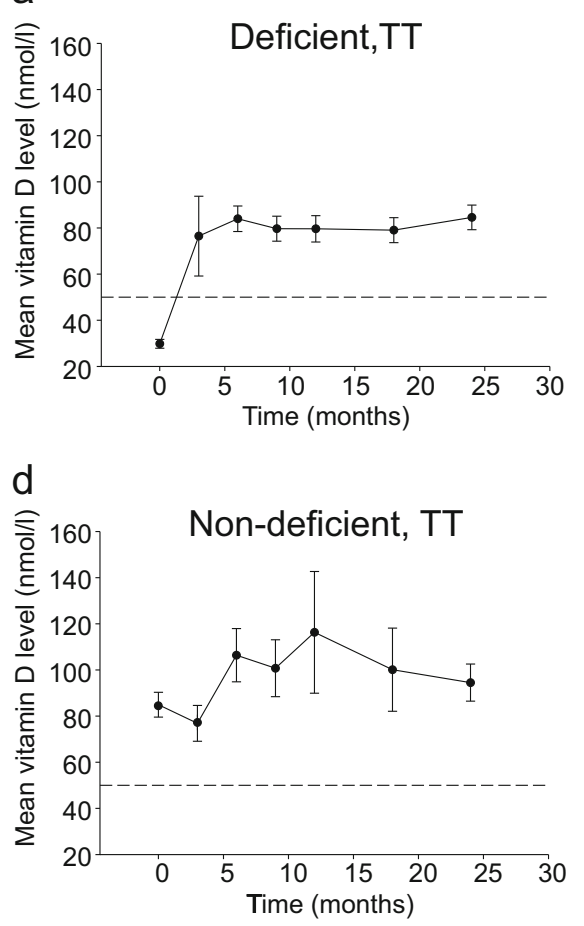

b

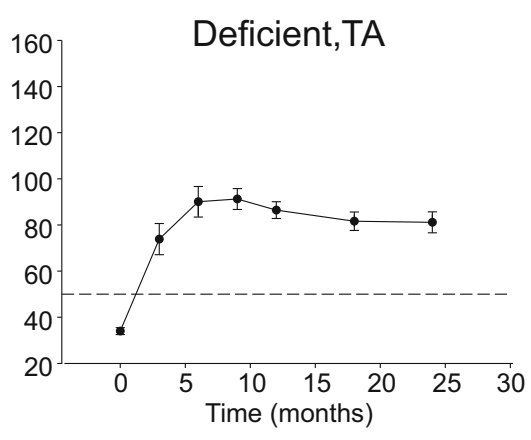

e

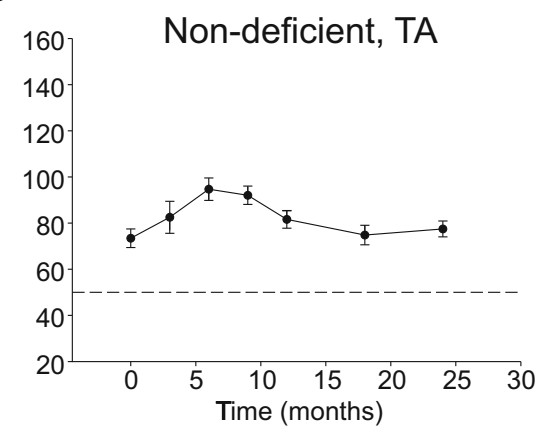

C

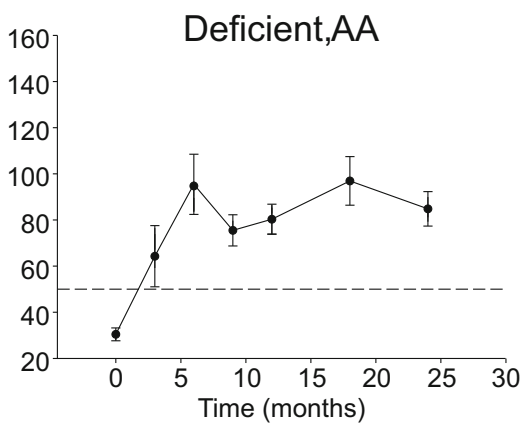

f

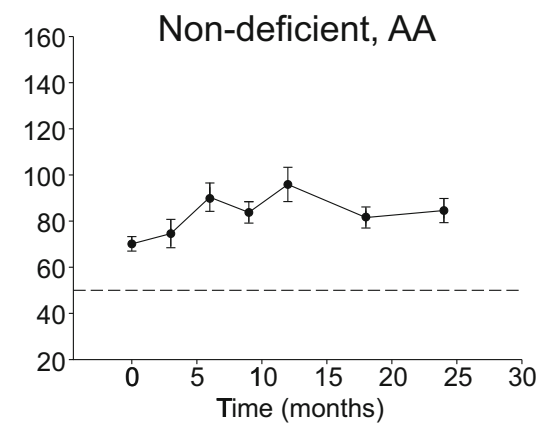

Fig. 2 Serum levels of 25-hydroxyvitamin D3 in patients before and after RYGB surgery, split by FTO rs 9939609 genotype and presurgery vitamin D status. Note that post-surgery, all patients received standard oral vitamin D3 supplements (1,200 IU/day). If serum levels of 25- hydroxyvitamin D3 were below $50 \mathrm{nmol} / \mathrm{L}$ (dashed lines) at follow-up investigations (i.e., at $+3,+6,+9,+12,+18$, and +24 months), patients received additional intramuscular injection of 300,000 IU every 3 months. Data are shown as mean \pm SEM 
carriers of the rs9939609 A-allele lost more body weight than noncarriers during the intervention period [14]. However, there are also studies that failed to show an impact of FTO on weight loss response to dietary interventions. For instance, in one study involving 204 Japanese women, FTO rs9939609 did not significantly change body weight or metabolic risk factors in response to calorie restriction [15].

The main finding of our study is that patients with a preoperative vitamin $\mathrm{D}$ deficiency lost more weight following RYGB surgery when they carried two copies of FTO rs9939609 A-allele, than their homozygote counterparts did, i.e., TT carriers who had a vitamin D deficiency. If replicated by others, these findings suggest that the effect of FTO on body weight regulation in humans is modulated by vitamin D. In line with our observation, a previous genetic study demonstrated that children who carried the FTO A-allele showed a greater weight gain over 5 years compared to noncarriers, however, only in those who were vitamin D-deficient [5]. This suggests that vitamin D may possess biological properties that can regulate the magnitude by which FTO or its genetic network [16, 17] impacts body weight regulation in humans. Vitamin D fulfills many biological functions in humans, comprising whole-body calcium metabolism [18]. A recent study has shown that FTO interacts with three isoforms of calciumdependent protein kinase II: $\alpha, \beta$, and $\gamma$. These protein kinases phosphorylate a broad range of substrates, including factors involved in the regulation of food intake and energy homeostasis (e.g., BDNF, NPY1R) [19]. With this in mind, it could be speculated that FTO A-allele carriers who were deficient for vitamin D before RYGB surgery (a condition that is expected to concur with a low calcium bioavailability) showed the greatest weight loss after this procedure because vitamin D supplementation exerted the strongest restorative effect on mineral homeostasis in this genotype group. In addition to its effect on calcium homeostasis, vitamin $\mathrm{D}$ regulates the transcription of numerous genes by activation of the nuclear vitamin D receptor [18]. Thus, it could be speculated that post-operative vitamin $\mathrm{D}$ supplementation regulated either the transcription of FTO-which encodes a $\mathrm{N}^{6}$-RNA demethylase [20] — or the transcription of genes that are functionally connected with FTO (e.g. IRX3 or RPGRIPIL), or both, and that these effects of post-operative vitamin D supplementation on genetic regulation are strongest in vitamin Ddeficient FTO A-allele carriers. However, the reader must keep in mind that these explanations are speculative and require further study.

Vitamin D can be synthesized by the human skin in response to sunlight exposure. Thus, vitamin D deficiency could also be a result of an inactive lifestyle, hallmarked by sedentarism and home-based activities (e.g., TV watching) [21-24]. Interestingly, a previous study involving patients who underwent bypass surgery has shown that patients significantly increase the time spent in leisure activities after the surgery [25]. With this in mind, it could be argued that RYGB surgery may have increased the time spent in leisure activities especially in those who were most sedentary before the surgery, i.e., obese patients who were vitamin D-deficient. Of note, physical activity has been shown to counteract the impact of FTO on body weight in humans [26]. In the same way, physical activity may boost the weight loss after RYGB surgery in AA carriers. However, bearing in mind that our study was of correlative nature, caution is needed before any firm conclusion can be drawn about the mechanism through which vitamin D may influence the impact of FTO on weight dynamics in humans.

As of yet, there is not much evidence for a beneficial effect of vitamin D supplementation on body weight regulation in humans. In a series of randomized controlled human studies on vitamin D supplementation, only one involving 36,282 post-menopausal women demonstrated that the supplementation of calcium plus vitamin $\mathrm{D}$ had a minimal but consistent favorable effect on weight (reviewed in [27]). However, these studies did not control for FTO [27], which may explain why the majority failed to show any beneficial effects of vitamin D supplementation on weight. Alternatively, the putative lack of efficacy of vitamin D supplementation in body weight reduction may also hint to vitamin D status as a simple marker of a sedentary lifestyle. This notion would also be in accordance with the previously found association of vitamin D deficiency and metabolic disease like type 2 diabetes [28, 29].

\section{Strengths and Limitations}

Strengths of this study are that the study cohort was treated in a highly standardized fashion in a single center, thereby largely eliminating the biasing influence of distinct post-surgical care. Furthermore, since respective data were systematically collected, all analyses in our study could be adjusted for potential confounders such as age, sex, BMI before surgery, specific type of surgery, and season of vitamin measurement. However, also several limitations of our study need to be stated. Due to the 2-year follow-up time of our study, it remains unclear whether the observed interplay of FTO and vitamin D with weight loss persists over a longer follow-up period. Another limitation is that possible confounds by other factors, such as physical activity and the interplay of FTO with other genes [30,31], which were not considered in the present analysis, cannot be excluded. Finally, generalization of our findings to other age groups (e.g., obese children undergoing bariatric surgery) or ethnic groups may not be appropriate.

\section{Conclusions}

Our study suggests that the presurgery vitamin D status of patients undergoing RYGB surgery might be crucial for 
subsequent weight loss, especially in those who carry two copies of the FTO rs9939609 A-allele. Our findings could also offer a possible explanation as to why previous studies investigating the link between FTO and weight dynamics in humans have produced somewhat inconsistent results $[6,14$, 15], as the vitamin D status of participants before starting the weight loss intervention may have not been taken into account in these studies. Supporting this view, in our cohort, nearly half of the obese patients exhibited a vitamin D deficiency before surgery, demonstrating that this micronutrient deficiency is abundant in obese people [32, 33].

Acknowledgments The study was supported by the Swedish Research Council, the Swedish Brain Research Foundation, and the Novo Nordisk Foundation. We thank all of our patients that provided the blood samples and clinical information. Genotyping was performed by the SNP\&SEQ Technology Platform in Uppsala. The platform is part of Science for Life Laboratory at Uppsala University and supported as a national infrastructure by the Swedish Research Council.

Conflict of Interest MB, BS, BE, MT, HBS, and CB declare no conflicts of interest. The study was performed according to the 1964 Declaration of Helsinki and its later amendments. All patients provided written informed consent to the scientific use of their clinical data sets and blood samples for genetic analyses, and the study protocol was approved by the local ethic committee of the Canton St. Gallen.

Author Contribution BS, MT, and BE designed the study; BE and BS wrote the protocol; BS, MT, and BE collected the data; MB conducted the analyses; and all authors contributed to writing. All authors have approved the final manuscript.

Funding The study was supported by the Swedish Research Council (HBS), the Swedish Brain Research Foundation (CB and HBS), and the Novo Nordisk Foundation (CB).

Open Access This article is distributed under the terms of the Creative Commons Attribution License which permits any use, distribution, and reproduction in any medium, provided the original author(s) and the source are credited.

\section{References}

1. Cornelis MC, Qi L, Zhang C, et al. Joint effects of common genetic variants on the risk for type 2 diabetes in U.S. men and women of European ancestry. Ann Intern Med. 2009;150(8):541-50.

2. Elks CE, Perry JR, Sulem P, et al. Thirty new loci for age at menarche identified by a meta-analysis of genome-wide association studies. Nat Genet. 2010;42:1077-85.

3. Peng S, Zhu Y, Xu F, et al. FTO gene polymorphisms and obesity risk: a meta-analysis. BMC Med. 2011;9:71.

4. Li H, Kilpeläinen TO, Liu C, et al. Association of genetic variation in FTO with risk of obesity and type 2 diabetes with data from 96,551 East and South Asians. Diabetologia. 2012;55:981-95.

5. Lourenço BH, Qi L, Willett WC, et al. FTO Genotype, Vitamin D status, and weight gain during childhood. Diabetes. 2014;63:808-14.

6. Liou T-HH, Chen H-HH, Wang W, et al. ESR1, FTO, and UCP2 genes interact with bariatric surgery affecting weight loss and glycemic control in severely obese patients. Obesity Surg. 2011;21:175865 .
7. Still CD, Wood GC, Chu X, et al. Clinical factors associated with weight loss outcomes after Roux-en-Y gastric bypass surgery. Obesity (Silver Spring). 2014;22:888-94.

8. Mägi R, Manning S, Yousseif A, et al. Contribution of 32 GWASidentified common variants to severe obesity in European adults referred for bariatric surgery. PLoS One. 2013;8:e70735.

9. Thurnheer M, Bisang P, Ernst B, et al. A novel distal very long Rouxen Y gastric bypass (DVLRYGB) as a primary bariatric procedurecomplication rates, weight loss, and nutritional/metabolic changes in the first 355 patients. Obesity Surg. 2012;22:1427-36.

10. Gröber U, Spitz J, Reichrath J, et al. Vitamin D: update 2013: from rickets prophylaxis to general preventive healthcare. Dermatoendocrinol. 2013;5:331-47.

11. Ernst B, Thurnheer M, Schmid SM, et al. Seasonal variation in the deficiency of 25-hydroxyvitamin D(3) in mildly to extremely obese subjects. Obesity Surg. 2009;19:180-3.

12. Deitel M, Greenstein RJ. Recommendations for reporting weight loss. Obes Surg. 2003;13(2):159-60.

13. Loos RJ. Recent progress in the genetics of common obesity. Br J Clin Pharmacol. 2009;68:811-29.

14. de Luis DA, Aller R, Conde R, et al. The rs 9939609 gene variant in FTO modified the metabolic response of weight loss after a 3-month intervention with a hypocaloric diet. J Investig Med. 2013;61:22-6.

15. Matsuo T, Nakata Y, Murotake Y, et al. Effects of FTO genotype on weight loss and metabolic risk factors in response to calorie restriction among Japanese women. Obesity (Silver Spring). 2012;20: $1122-6$.

16. Smemo S, Tena JJ, Kim K-HH, et al. Obesity-associated variants within FTO form long-range functional connections with IRX3. Nature. 2014;507:371-5.

17. Stratigopoulos G, Carli J, O’Day D, et al. Hypomorphism for RPGR IP1L, a ciliary gene vicinal to the FTO locus, causes increased adiposity in mice. Cell Metab. 2014;19:767779.

18. Dusso AS, Brown AJ, Slatopolsky E. Vitamin D. Am J Physiol Renal Physiol. 2005;289:F8-28.

19. Lin L, Hales CM, Garber K, et al. Fat mass and obesity-associated (FTO) protein interacts with CaMKII and modulates the activity of CREB signaling pathway. Hum Mol Genet. 2014;23:3299-306.

20. Jia G, Fu Y, Zhao X, et al. N6-methyladenosine in nuclear RNA is a major substrate of the obesity-associated FTO. Nat Chem Biol. 2011;7:885-7.

21. Lips P, van Schoor NM, de Jongh RT. Diet, sun, and lifestyle as determinants of vitamin D status. Ann N Y Acad Sci. 2014;1317: 92-8.

22. Heuvel E, Schoor N, Jongh R, et al. Cross-sectional study on different characteristics of physical activity as determinants of vitamin D status; inadequate in half of the population. Eur J Clin Nutr. 2013;67(4): $360-5$.

23. Ahern T, Khattak A, O'Malley E, et al. Association between vitamin D status and physical function in the severely obese. J Clin Endocrinol Metab. 2014;99(7):E1327-31.

24. Larose TL, Chen Y, Camargo CA, et al. Factors associated with vitamin D deficiency in a Norwegian population: the HUNT Study. J Epidemiol Community Health. 2014;68:165-70.

25. Vatier C, Henegar C, Ciangura C, et al. Dynamic relations between sedentary behavior, physical activity, and body composition after bariatric surgery. Obesity Surg. 2012;22:1251-6.

26. Loos RJ, Yeo GS. The bigger picture of FTO - the first GWASidentified obesity gene. Nat Rev Endocrinol. 2014;10(1):51-61.

27. Song Q, Sergeev IN. Calcium and vitamin D in obesity. Nutr Res Rev. 2012;25:130-41.

28. Choi EY. 25(OH)D status and demographic and lifestyle determinants of $25(\mathrm{OH}) \mathrm{D}$ among Korean adults. Asia Pac J Clin Nutr. 2012;21:526-35. 
29. Thuesen B, Husemoen L, Fenger M, et al. Determinants of vitamin D status in a general population of Danish adults. Bone. 2012;50:60510

30. Harbron J, van der Merwe L, Zaahl MG, et al. Fat mass and obesityassociated (FTO) gene polymorphisms are associated with physical activity, food intake, eating behaviors, psychological health, and modeled change in body mass index in overweight/obese Caucasian adults. Nutrients. 2014;6:3130-52.
31. Gustavsson J, Mehlig K, Leander K, et al. FTO genotype, physical activity, and coronary heart disease risk in Swedish men and women. Circ Cardiovasc Genet. 2014;7:171-7.

32. Liel Y, Ulmer E, Shary J, et al. Low circulating vitamin D in obesity. Calcif Tissue Int. 1988;43:199-201.

33. Wortsman J, Matsuoka LY, Chen TC, et al. Decreased bioavailability of vitamin D in obesity. Am J Clin Nutr. 2000;72:690-3. 